\title{
IPADS, IPHONES, ANDROIDS, AND SMARTPHONES: FDA \\ Regulation of Mobile Phone Applications as MEDICAL DEVICES
}

\begin{tabular}{|c|c|}
\hline \multicolumn{2}{|r|}{ Alex Krouse } \\
\hline I. & INTRODUCTION \\
\hline \multirow[t]{4}{*}{ II. } & BACKGROUND \\
\hline & 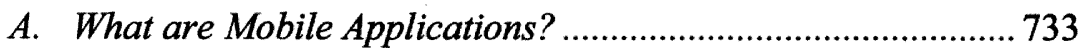 \\
\hline & B. Mobile Applications: A Brief History ................................. 733 \\
\hline & C. Mobile Application Uses and Complexities........................... 735 \\
\hline \multirow[t]{2}{*}{ III. } & MOBILE HEALTH \\
\hline & A. Mobile Health Industry ....................................... \\
\hline \multirow[t]{4}{*}{ IV. } & 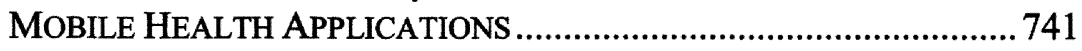 \\
\hline & A. Introduction to Mobile Health Applications ........................... 741 \\
\hline & B. Informational Mobile Health Applications ........................... 741 \\
\hline & C. Diagnosing Mobile Health Applications.............................. 743 \\
\hline \multirow[t]{4}{*}{ V. } & 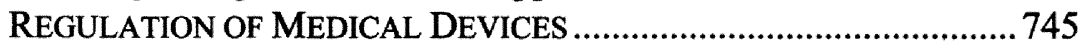 \\
\hline & A. Medical Device Definition by FDA .................................. 745 \\
\hline & B. Classes of Medical Devices Under the FDA.......................... 746 \\
\hline & C. Component vs. Accessory Regulation .................................... 748 \\
\hline \multirow[t]{4}{*}{ VI. } & ARE MOBILE APPLICATIONS MEDICAL DEVICES? ....................... 749 \\
\hline & 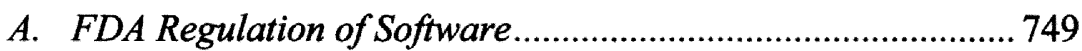 \\
\hline & B. Bearing the Burden of Regulation ..................................... 752 \\
\hline & C. Current FDA Approved Mobile Applications ......................... 753 \\
\hline \multirow[t]{4}{*}{ VII. } & REGULATORY SOLUTIONS FOR MOBILE HEALTH APPLICATIONS .. 755 \\
\hline & A. Defining Non-Regulated Mobile Health Applications ............ 756 \\
\hline & B. Defining Regulated Mobile Health Applications ................... 757 \\
\hline & C. Current FDA Position on Medical Device Review................. 757 \\
\hline \multirow[t]{4}{*}{ VIII. } & FAST TRACK SOLUTION FOR MOBILE HEALTH ............................760 \\
\hline & 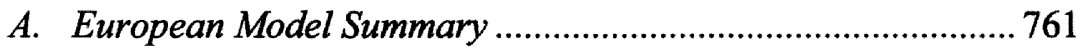 \\
\hline & B. Benefits of Separate Regulatory Scheme .............................. 762 \\
\hline & C. A Test Model for Mobile Health Applications ........................ 762 \\
\hline & CONCLUSION \\
\hline
\end{tabular}

\section{INTRODUCTION}

A person diagnosed with a form of arrhythmia, or abnormal electrical activity in the heart, decides to download the application Instant Heart Rate for their mobile smartphone. The person, knowing they have this condition,

* Alex Krouse, J.D., 2012, Indiana University Robert H. McKinney School of Law; M.H.A., 2012, Indiana University School of Medicine; B.A., 2008, DePauw University. 
would like to monitor their heart rate to make sure nothing is wrong. After discovering he or she can use their phone to check his or her heart rate, the person is thrilled. However, after using the application for a few weeks the person notices considerable differences between the phone application and other heart rate monitors. Luckily, this person noticed these differences because it is likely that without accurate readings, the person could put his or herself at risk of suffering a heart attack. To date, the Instant Heart Rate application has over 4 million downloads and has been rated by over $60,000{ }^{1}$

Mobile phone applications, or mobile applications, ${ }^{2}$ such as the one described above are becoming more prevalent every day. In particular, mobile health applications are seeing an increasing usage among smartphone ${ }^{3}$ users. People who carry smartphones can access information ranging from the local news to vital health and treatment facts. In addition, these applications do much more than simply allow the person to read information. For example, the Instant Heart Rate application for Android phones tells the user to "place the tip of your finger on the camera lens so it completely covers the lens. Hold your phone steady." Then, your current heart rate will be shown on the display. ${ }^{5}$ A user simply needs to download the application, and they will have a tool to monitor their heart rate. This might appear to be a minor advancement, but it highlights that mobile phone users are now beginning to rely on their smartphones for health purposes.

This Note addresses the major crossroads of regulation that mobile health applications are currently facing from the Food and Drug Administration ("FDA"). First, the mobile health industry is growing at an impressive rate, placing useful health applications at the fingertips of users daily. ${ }^{6}$ Second, the FDA has begun regulating mobile health applications as

1. Instant Heart Rate, APPBRAIN, http://www.appbrain.com/app/instant-heartrate/si.modula.android.instantheartrate (last visited Jan. 16, 2011).

2. A mobile phone application is "a term used to describe internet applications that run on smartphones and other mobile devices. Mobile applications usually help users by connecting them to internet services more commonly accessed on desktop or notebook computers, or help them by making it easier to use the internet on their portable devices." Mobile Application, WEBOPEDIA, http://www.webopedia.com/TERM/M/mobile_application. html (last visited Feb. 28, 2012).

3. Smartphone is defined as: "A cellular telephone with built-in applications and Internet access. Smartphones provide digital voice service as well as text messaging, e-mail, Web browsing, still and video cameras, MP3 player, video viewing and often video calling. In addition to their built-in functions, smartphones can run myriad applications, turning the once single-minded cellphone into a mobile computer." Definition of Smartphone, PCMAG.COM, http://www.pcmag.com/encyclopedia_term/0,2542,t=Smartphone\&i=51537, 00.asp (last visited Feb. 28, 2012).

4. APPBRAIN, supra note 1.

5. Id.

6. See, e.g., Chris Gullo, Health Apps Already a Bigger Market Than Remote Monitoring, MOBIHEALTHNews (Jan. 6, 2012), http://mobihealthnews.com/15646/health-appsalready-a-bigger-market-than-remote-monitoring/. 
medical devices. ${ }^{7}$ However, current FDA regulations for medical devices lack clarity regarding this new technology. Finally, due to the lack of clarity, the FDA must look to improve the regulatory structure to maintain a balance between product safety and innovation for mobile health applications. The increasing regularity of mobile device and mobile application use throughout society demonstrates the pressing importance of this issue. By implementing a separate regulatory scheme for mobile health applications, the FDA can address these concerns.

\section{BACKGROUND}

\section{A. What are Mobile Applications?}

A mobile application is a piece of software that is contained within a phone for a particular purpose or use. The purpose of mobile applications varies from providing information to actual interaction with the user. Essentially, the main goal for all mobile applications is to provide a service that can be used on cell phones rather than an actual computer. Although mobile applications are complex and serve various purposes, all share the ability of allowing the user to access information through this mobile device or cell phone. ${ }^{8}$

\section{B. Mobile Applications: A Brief History}

Mobile applications are a recent phenomenon, however not quite as recent as one may think. Although the mobile application industry has seen an increase since the advent of smartphones, mobile applications for mobile devices were developed as early as $1992 .{ }^{9}$ Examples of some early applications developed include calendars, lists, and other task oriented applications. Generally, these applications allowed users to keep track of information with their mobile phones. This increased mobility of information allowed users to save time, money, and confusion by providing access to the information at all times. However, the true potential of mobile phone applications was not evident until the recently.

Due to technological innovations, mobile devices ${ }^{10}$ are now able to

7. Draft Guidance for Industry and Food and Drug Administration Staff - Mobile Medical Applications, U.S. FOOD \& DRUG ADMIN. (July 21, 2011), http://www.fda.gov/ MedicalDevices/DeviceRegulationandGuidance/GuidanceDocuments/ucm263280.htm.

8. WEBOPEDIA, supra note 2.

9. Smartphone: Access or Excess, ARLINGTONIAN.COM (Oct. 6, 2010), http://www.arlingtonian.com/archives/2628; see also John Schneidawind, Poindexter putting finger on PC bugs, USA TODAY, Nov. 23, 1992, at 2B.

10. A mobile device is defined as: a computing device that is typically smaller than a computer, however still has the capability to contain a keyboard or touchscreen for user input. Examples include the iPhone, iPad, Blackberry devices, Android devices, and tablet 
view websites and data through the various cellular networks. These innovations allow developers of software to build programs specified for mobile devices. Initially, the need for various applications was quite low. The smartphone market only truly began in 1997 with the Ericsson GS88. ${ }^{11}$ In addition, the widely popular Blackberry phone did not attain widespread popularity until the early 21 st century. ${ }^{12}$ The true advent of mobile applications occurred shortly after Apple Inc. developed the iPhone, which was released in the summer of 2007. ${ }^{13}$ That summer, Apple stated that the $\mathrm{iPh}$ one could support applications built by third parties. ${ }^{14}$ This development led to the use of online application stores which further enabled users to use mobile applications on their cellular devices.

The increased use of mobile applications is the result of an increase in mobile device use and the development of online application stores. The online application stores create an environment for third party developers to sell their applications which can then be used on mobile devices that use the appropriate operating system. ${ }^{15}$ These stores have now become commonplace across mobile phone networks. They have been created by Apple for the iPhone, Blackberry for Blackberry devices, and Android for mobile phones using the Android operating system.

One of the most popular online stores is the Apple App Store. With the development of the iPhone, the application store allowed the masses to develop applications. Currently, over 350,000 applications are available through the Apple App Store. ${ }^{16}$ Another influential online application store is called the Android Market. This online store is developed on the Google operating system known as Android, where the applications are compatible with Android operating mobile devices. Currently the store contains over 100,000 applications. ${ }^{17}$ Finally, the Blackberry App World is another store online designed for application sales. The Blackberry App World contained

computers. See Priya Viswanathan, What is a Mobile Device?, ABOUT.coM, $\mathrm{http}: / /$ mobiledevices.about.com/od/glossary/g/What-Is-A-Mobile-Device.htm (last visited Mar. 10, 2012).

11. History, STOCKHOLM SMARTPHONE, http://www.stockholmsmartphone.org/history/ (last visited Feb. 28, 2012).

12. The History of the Blackberry, BBGEEKS (Apr. 15, 2008), http://www.bbgeeks. com/blackberry-guides/the-history-of-the-blackberry- $88296 /$.

13. iPhone $2 \mathrm{G}$ History, IPHONE HISTORY, http://www.iphonehistory:com/iphone-2g/ (last visited Feb. 28, 2012).

14. Press Release, Apple Inc., iPhone to Support Third-Party Web 2.0 Applications (Jan. 11, 2007), http://www.apple.com/pr/library/2007/06/1 liphone.html.

15. Operating system is defined as: "software that controls the execution of computer programs and may provide various services." Definition of Operating System, WORDNET SEARCH - 3.1, http://wordnetweb.princeton.edu/perl/webwn (last visited Mar. 10, 2012) (type "Operating System" in box labeled "Word to search for;" then click "Search WordNet").

16. Christian Zibreg, When Will Android People Pay for Quality?, 9T05MAC (Mar. 10, 2011, 12:20 PM), http://www.9to5mac.com/55273/when-will-android-people-pay-forquality/.

17. Edwin Kee, Google Android Hits 100,000 Application Milestone on Android Market, UBERGIzMO (Oct. 25, 2010, 9:41 PM), http://www.ubergizmo.com/15/archives/2010/ 10/google_android_hits_100000_application_milestone_on_android_market.html. 
over 10,000 applications as of September $2010 .{ }^{18}$ All of these application stores have allowed users the ultimate access to data through their mobile devices.

The most important innovation in the development of the mobile application industry is that the applications can be used on multiple devices beyond cellular telephones. For example, an application downloaded off of the Apple App Store likely works on not only the iPhone but also the iPad tablet and the iTouch mp3 player. This is due to the fact that the applications are built on the specific operating platforms that are used by individual mobile devices. This also occurs with both Android and Blackberry devices. The latter two companies have their operating systems on tablets similar to the iPad, which is important because like Apple Inc. these applications are not only able to be used on cellular devices but also other mobile devices that are not used as cell phones. The transferability of these applications makes regulating mobile applications a growing problem.

\section{Mobile Application Uses and Complexities}

Mobile phone applications have created a new industry within the world. For the first time, consumers are able to use their phones as more than a telephone. Also, because these applications can be used across multiple devices, the technology industry has developed mobile phones that are similar to computers and computers that are similar to mobile phones. A prime example of this is the iPad. The overall purpose of these applications cannot be easily defined; however, it is necessary to understand the true complexities. Some simply allow the user to read information, while others almost perform a task for the user that could not be otherwise done without the application.

The most effective way to understand mobile applications is through examples. First, there are some common mobile applications that allow a user to use the Internet easier or to help read information from a mobile device. A prime example is the mobile browser Skyfire. This application allows users to download the software onto their device and read webpages similarly to the way one would read or use a website on their computer. ${ }^{19}$ Because it is tough to read or view an entire webpage from a mobile phone, Skyfire is an application that makes this transition easier for the user by

18. Donald Melanson, Blackberry App World Crosses the 10,000 Mark, ENGADGET (Sept. 8, 2010, 10:53 PM), http://www.engadget.com/2010/09/08/blackberry-app-worldcrosses-the-10-000-app-mark/.

19. Skyfire mobile browser improves the mobile Internet experience by allowing users to read webpages in a similar format to computers. Not all webpages transition easily to smaller screens used on mobile devices, and this application makes this transition to smaller screens. See Cloud Solutions for Mobility, SKYFIRE.COM, http://www.skyfire.com/ (last visited Feb. 20, 2012). 
running the webpage on a server and relaying it to the end user. ${ }^{20}$ However, applications such as Skyfire are just the beginning. Considering the number of applications available, this is not surprising. Another example is the application Google Maps, developed by Google Inc., which allows users to obtain directions or even locate their specific position within the map. ${ }^{21}$ The user, if other friends have the same application, can even see the location of a friend within the map and get directions specifically to the location of the other person's device. ${ }^{22}$

Mobile applications are also used for different purposes beyond location and making one's device more efficient for Internet use, such as the Shop Savvy application, which allows the user to download the application for the purpose of shopping. ${ }^{23}$ The application gives the user the ability to scan barcodes of products in stores and compare prices to other stores in the area or online. ${ }^{24}$ Obviously, without the application shoppers would need to scan the barcode at the register within the store to find the price. This is one example of a common application, offering a complex yet new way to shop and save time.

Thus far, the applications discussed have involved common applications that are geared towards the general public. However, there are a growing number of applications geared toward specific industries and professionals, including medicine. These types of applications highlight the complexities involved in developing mobile applications.

Industry specific applications are not reaching out to the general public but are specifying their application for the needs of enterprises or corporations. $^{25}$ This category would also include applications for individuals within specific professions or jobs. These types of applications are complex in a different way: they offer professional knowledge to professionals in a given field if downloaded and used. An industry specific application includes the Concrete Calc, which allows users to find the cubic yards of

20. Id.

21. See Google Maps for Mobile, GoOGLE MOBILE, http:/www.google.com/mobile/ maps/ (last visited Feb. 20, 2012).

22. See Google Latitude, GoOGLE MOBILE, http:/www.google.com/mobile/latitude/ (last visited Feb. 20, 2012).

23. See Shop Savvy (Barcode Scanner and $Q R$ Code Reader), ITUNES PREVIEW, http://itunes.apple.com/us/app/shop-savvy-barcode-scanner/id338828953?mt $=8$ (last visited Feb. 20, 2012).

24. Id.

25. Industry specific applications are currently being built for corporations to make their own internal processes easier. One example is a company called Rehabcare, which uses mobile applications to facilitate its therapy services. Nancy Gohring, Cloud Services Spur Mobile Enterprise Apps, PCWORLD, http://www.pcworld.com/article/209072/ cloud_services_spur_mobile_enterprise_apps.html (last visited Feb. 20, 2012). "The therapists use the app to view their schedules, look up patient information and start and stop their time clocks. By the end of the year, Rehabcare hopes to have deployed 8,000 iPod Touches so that therapists can use the application." Id. 
concrete needed for a specific job. ${ }^{26}$ Although it appears to be a basic calculator, the application page explains that it is "good for building, construction or DIY."27 This description highlights that the application developer intends this calculator to be used for building on a novice to expert level.

Another example of a more complex industry specific mobile application is the cadTouch R2. This application "allows users to draw floorplans, land surfaces (and calculate area and perimeter), facades, [and] mechanical or structural parts." ${ }^{28}$ Applications such as these are geared towards construction managers, engineers, and other builders. Obviously the application provides an easier method to figure out complex problems with construction. Finally, iHydrate, developed by Mobile Sports Inc., is geared towards coaches or even parents of athletes. ${ }^{29}$ The application allows the user to see the current temperature at their location, displays the risk of heat illness, and allows the user to schedule hydration reminders. ${ }^{30}$

By understanding some examples of industry specific applications, it becomes easier to see what developers are trying to do with mobile applications for devices. Developers are attempting to bring the resources that consumers have at a computer to handheld devices at all times, which has many consequences for the health care industry. Some applications can be developed strictly for professionals, while others can be easily accessed by the general public. Because mobile applications are being developed for health care providers every day, numerous issues regarding to regulation are created. These applications might be solely informational, but many are also geared towards diagnosing and treating patients, which highlights the ultimate risk. ${ }^{31}$

\section{MoBILE HEALTH}

\section{A. Mobile Health Industry}

mHealth, also known as mobile health, is a term used for "the delivery

26. See Concrete Calc, ITUNES PREVIEw, http://itunes.apple.com/us/app/concretecalculator/id317133083? $\mathrm{mt}=8$ (last visited Feb. 20, 2012).

27. $I d$.

28. Houston Neal, The Best Construction Management Apps for the iPhone and iPad, SOFTWARE ADVICE, http://www.softwareadvice.com/articles/construction/the-best-constr uction-management-apps-for-the-iphone-and-ipad-1082510/ (last visited Feb. 20, 2012).

29. iPhone App Helps Keep Athletes Well Hydrated, Avoid Heat-Related Illness, MOMSTEAM, http://www.momsteam.com/health-safety/ihydrate-application-helps-athletesstay-hydrated (last visited Feb. 20, 2012).

30. Id.

31. ResolutionMD Mobile allows doctors to send images to other mobile devices such as the iPhone or Android smartphone for viewing and diagnostic purposes. See Cindy Waxer, How Mayo Clinic Doctors Use Smartphones to Diagnose Patients, ITwORLD (Oct. 28, 2010, 8:43 PM), http://www.itworld.com/software/125744/how-mayo-clinic-doctors-usesmartphones-diagnose-patients. 
of healthcare services via mobile communication devices.,32 There are other definitions, but, overall, mHealth is a term used for the practice of clinical medicine and public health supported by mobile devices. ${ }^{33}$ Mobile health applications play a pivotal role in the mHealth movement because mHealth relies on providers' use of these applications when servicing patients. This creates a dilemma that has not been seen since the development of computers. Providers have been using software to aid their medical decisions for some time, but now providers can also use their mobile devices to help them make actual diagnoses. In addition, mHealth is offering solutions directly to the consumer. While in developing countries this mobility is seen as a major innovation, in highly regulated countries, such as the United States, this can create problems. Even though the industry is still in its infancy, statistics show that mHealth is growing exponentially.

The data discussed previously highlights the influence of mobile applications as a whole; however, recent information also shows that mobile applications are transitioning into the health care industry. According to a survey by CTIA and Harris Interactive, seventy-eight percent of the United States is interested in mobile health solutions. ${ }^{34}$ It is important to understand the general industry and movement in order to understand how important regulation of this industry may be. To highlight this, twenty-three percent of the responders believed that mobile health could replace doctor visits altogether. ${ }^{35}$ If consumers will use mobile health applications in lieu of medical professional visits, the need for regulation is apparent. This need is ultimately in a grey area, which needs to be addressed.

The grey area that has developed creates unprecedented consumer and professional access to mobile applications, some of which should be used strictly by professionals. Access to wireless health solutions in the United States is impressive. Currently, almost ninety percent of the U.S. population has a mobile phone. ${ }^{36}$ In addition, close to nineteen percent of people have smartphones that are capable of using the most current mobile health applications. ${ }^{37}$ Obviously consumers have access to mobile applications; but, medical professionals themselves are extremely important to delivering care through technology. This creates a dilemma between increasing access to mobile health solutions and the risk of consumers using these applications for diagnosing and treatment.

Providers themselves are contributing to this dilemma because there

32. Carol Torgan, The mHealth Summit: Local \& Global Converge, KINETICs (Nov. 6, 2009), http://www.caroltorgan.com/mhealth-summit/.

33. See id.

34. MobiHealthNews, Wireless Health: State of THE INDUStry 2009 YeAR END REPORT 1, 2 (2009), available at http://mobihealthnews.com/wp-content/Reports/2009 StateoftheIndustry.pdf.

35. Id. at 2-3.

36. Id. at 4.

37. Id at 5 . 
are definite cost, time and effort benefits created through this new mobile health industry. "Manhattan Research found that 64 percent of physicians use a smartphone today. That's 20 percent more physicians than in 2008 . By 2013,81 percent of physicians will use smartphones." 38 While the industry has not yet fully adjusted to the influx of these applications, the future statistics highlight that mobile health capabilities will increase exponentially. Physicians and nurses will be able to make decisions in a faster and more efficient manner. Nonetheless, the costs savings possible show the dilemma regulatory agencies have regarding mobile applications.

During a time when the United States spends $16.7 \%$ of its GDP on health care, the use of mobile health has the potential to be a tool in reducing cost and promoting efficiencies in health care delivery. ${ }^{39}$ In 1990, the United States spent $\$ 714$ billion on health care, but by 2008 the United States had spent over $\$ 2.3$ trillion on health care. ${ }^{40}$ These startling statistics demonstrate the need for more effective use of technology to promote efficiencies and cost savings. As an example of the cost saving potential, Verizon Wireless estimates that their mobile broadband solutions saved nearly $\$ 6.9$ billion through improved productivity, and " $[t]$ hat figure is expected to increase to $\$ 27.2$ billion by $2016 .{ }^{, 41}$ Although this is one example, the possibilities resulting from increased technological access to hospitals and medical providers are endless.

The overall mobile health application industry has been expanding because of these catalysts. According to MobiHealthNews, as of March 2010, there were 6000 mobile health applications available online with nearly thirty percent intended for use by healthcare professionals. ${ }^{42}$ That means almost 2000 applications are being directly marketed towards medical professionals. ${ }^{43}$ Some of these applications cost as much as 299 dollars. $^{44}$ With numerous options in the industry's infancy, medical providers already have unprecedented access to mobile applications. Despite the mobile health application industry being in its infancy, medical providers have unprecedented access to mobile applications.

Not only are there many mobile health applications currently available on the online stores, their numbers are increasing at a rapid pace. As of September 2010, mobile health applications on the Apple AppStore have

38. Id.

39. U.S. Health Care Costs, KAISEREDU.ORG, http://www.kaiseredu.org/IssueModules/US-Health-Care-Costs/Background-Brief.aspx (last visited Oct. 31, 2010).

40. Id.

41. MobiHealthNews, supra note 38 , at 5 .

42. Brian Dolan, 3 Million Downloads for Android Health Apps, MoBIHEALTHNEws (Mar. 11, 2010), http://mobihealthnews.com/6908/3-million-downloads-for-android-healthapps/.

43. Id.

44. Id. 
grown by $66.6 \%$ within a 7 -month timeframe. ${ }^{45}$ During the same period, the number of mobile health applications available on the Android Market grew by $156.6 \%$ and by $141.4 \%$ in the Blackberry App World. ${ }^{46}$

Recognizing the increasing amount of mobile health applications available for download, it is necessary to analyze whether physicians are actually using applications for medical purposes. A report conducted by SDI found that ninety-five percent of the physicians who used mobile devices or smartphones utilize mobile health applications to access medical information. ${ }^{47}$ Because physicians are already using different forms of applications, the increase of applications will only increase their ability to utilize mobile application technology.

Another factor increasing the prevalence of mobile health applications has been the actions of large corporations targeting health care providers for this new technology, highlighting the need for more regulation of these mobile health applications. For example, AT\&T recently created an entire division devoted toward targeting the health care industry. ${ }^{48}$ One of their initial offerings is an "intelligent pill-bottle cap[]"" that sends a text message to caregivers when it is not opened on a daily basis. ${ }^{49}$ Although this is not an application itself developed by a third party, it does show that corporations are recognizing the strong need for mobile health solutions.

Other large companies have also invested heavily in mobile health technology. Verizon Wireless recently announced a deal with BL Healthcare for health applications that will work on BL's wireless devices. ${ }^{50}$ Additionally, Verizon has promoted applications for home health care workers and mobile video consultations. ${ }^{51}$ Even more interesting is Apple Inc.'s showcasing of medical applications to be used with their iPhone to physicians during workshops at its stores. ${ }^{52}$ Additionally, Apple has a designated space for medicine on their website which includes many of their technologies. ${ }^{53}$

These examples emphasize the need for further regulation because the

45. Dolan, supra note 45.

46. Id.

47. SDI Reports: Nearly a Third Of Physicians Use Handheld and Smartphone Devices to Access Medical Information, PRWEB (Mar. 4, 2010), http://www.prweb.com/releases/ SDI/SocialMediaStudy/prweb3681704.htm.

48. Peter Svensson, AT\&T Sets up Division to Target Health Care, WIRELESS WEEK (Nov. 5, 2010), http://www.wirelessweek.com/News/2010/11/Policy-and-Industry-ATTDivision-Target-Healthcare/.

49. Id.

50. Brian Dolan, Mobile Health 20/20: Verizon, Google, Glowcap, MoBiHEALTHNEws (Aug. 11, 2010), http://mobihealthnews.com/8605/mobile-health-2020verizon-google-glowcap/.

51. Id.

52. Iltifat Husain, Apple is Actively Recruiting Health Care Providers at their Retail Stores, IMEDICALAPPS (May 6, 2010), http://www.imedicalapps.com/2010/05/applehealthcare-providerselectronic-medical-records-ipad-iphone/.

53. Id. 
industry can and will expand into every facet of medical practice. Corporate movement into the arena of mobile health has spurred the development of applications, and as discussed above, some companies are even promoting the use of these applications. As mentioned previously, some applications are merely informational while others are extremely complex. Only by analyzing current mobile health applications is it possible to understand the dividing line of what applications should be regulated. However, a balance between innovation and regulation needs to be struck because of the extensive investment in the industry by mobile phone companies, third party developers, and medical providers.

\section{MOBILE HEALTH APPLICATIONS}

\section{A. Introduction to Mobile Health Applications}

Understanding the mobile health application industry is important in recognizing the delicate nature of these new innovations. On one hand, an entire new industry is being developed for people who have mobile devices or smartphones. Yet on the other hand, mobile health applications are giving unprecedented access to health diagnosis, treatment, and decisionmaking software to everyone with access. In order to fully understand the amount of regulation needed though, it is necessary to consider the types of applications offered and the level of medical intervention they provide.

Mobile health applications are surprisingly innovative and have truly amazing capabilities. There appear to be two main types of mobile health applications: information-based and diagnostic. When a mobile health application acts simply as an informational source, it provides little more than a website or a book; however, as the function of an application becomes more diagnostic the need for regulation becomes more realistic. By analyzing the currently developed models, a more firm dividing line can be discerned to decide what level of regulation is necessary for each individual mobile health application.

\section{B. Informational Mobile Health Applications}

Informational applications allow the user to read, input their own data, and gather resources to make decisions. Applications such as these have been used for quite some time, but they have mostly been utilized by medical professionals. One prime example of an information health application is Epocrates, which is an application built for iPhones, iPods, BlackBerrys, and Androids among others. ${ }^{54}$ Epocrates software allows the user to look

54. Mobile Products, EPOCRATES INC., http://www.epocrates.com/mobile (last visited Mar. 3, 2012). 
up drug information, coding references, and medical dictionary terms. ${ }^{55}$ As of 2010, Epocrates had one million users, including forty percent of physicians in the United States. ${ }^{56}$ This information is used by the physician to make medical decisions based on his or her own knowledge. However, use of this application provides a reference on hand at all times. Although this application is geared towards medical providers, anyone can download and use the application. With the current application explosion, informational applications are becoming more useful for the everyday user in addition to medical providers.

The Medscape app for the iPhone or iPod Touch is another application that allows the user to reference drugs, check drug interactions, find disease references, and view step by step instructions for clinical procedures. ${ }^{57}$ Medscape is similar to Epocrates in that its main purpose is for information rather than an actual medical procedure. Nonetheless, these two examples highlight informational mobile health applications predominately targeted towards medical providers, although consumers can still easily download these products.

In addition, other mobile health applications have been developed for the common consumer rather than a health care provider. An example of this type of mobile health application is the Let's Move It application. The Let's Move It mobile health application, developed by Cleveland Clinic Wellness Enterprise, is an application that allows the user to track steps taken, calories burned, weight loss, and allows the user to set goals for activity. ${ }^{58}$ This application is available for download on Apple devices as well as Android devices ${ }^{59}$ It provides information and feedback on the user's activity but is merely informational for the user, as it only offers a data tracking system. $^{60}$

Another example of a consumer focused mobile health application is the Cures A-Z application. Although this application is marketed toward the general consumer rather than a medical provider, the application developed by Plum Amazing purports to be a "free Comprehensive Medicine specialist in your pocket." 61 The actual application contains a complete list of health conditions and their treatments in order for users to manage an

55. Id.

56. Epocrates Files to Raise up to $\$ 75 M$ in IPO, S.F. BUS. TIMES (July 16, 2010), http://www.bizjournals.com/sanfrancisco/stories/2010/07/12/daily88.html.

57. Medscape Mobile, MEDSCAPE, http://www.medscape.com/public/iphone?src=ppc google\&ef_id=:20101104145047:s (last visited Nov. 4, 2010).

58. Let's Move It, ITUNES PREVIEW, http://itunes.apple.com/us/app/lets-move$\mathrm{it} / \mathrm{id} 396874560$ ? $\mathrm{mt}=8$ (last visited Mar. 10, 2012).

59. Id.

60. See id.

61. Cures $A-Z$, ITUNES PreviEw, http://itunes.apple.com/us/app/cures-a-z/id29764 8638 ? $\mathrm{mt}=8$ (last visited Mar. 10, 2012). 
illness. $^{62}$ Additionally, the application can teach the user about nutrition and the benefits of living a healthy lifestyle. ${ }^{63}$ Although this application is informational, it does purport to act as a medical professional in one's pocket. $^{64}$

The two previous examples demonstrate that mobile health applications once were more informational although some very noticeable grey areas existed. For example, with certain applications users can provide their own treatments. The user is given the information not just for his or her own knowledge but to actually diagnose his or her problem in hopes of finding treatment. However, obviously the information provided is similar to a website rather than an actual medical tool used for diagnosis. Nonetheless, these forms of applications are numerous and the dividing line between an informational application, and an application for diagnosis is hardly noticeable at all times.

\section{Diagnosing Mobile Health Applications}

Mobile health applications that are used in diagnosis and treatment are of the utmost concern because some allow decision processes normally handled by medical professionals to now be entirely dependent on the software. The technology used for mobile health applications is complex and the applications normally allow the user to solve an illness or act to give the user information regarding their illness. Currently, these mobile health applications are geared towards medical providers; however, there are many that everyday consumers could use for their own purposes. Although these applications are in their infancy, the industry has made impressive strides in developing mobile health applications that act beyond informational purposes.

A prime example of a diagnosing mobile health application is Instant Heart Rate. Instant Heart Rate is an application built for the iPhone and Android devices. ${ }^{65}$ The application can take the user's heart rate by allowing the user to place his or her finger over the camera for ten seconds. ${ }^{66}$ The developer explains that "it works by tracking the color changes in the light that passes through your finger" and acts "as a medical pulse oximeter but without a dedicated light source." ${ }^{, 67}$ Bear in mind, the FDA has drafted guidance on FDA approval of pulse oximeters. ${ }^{68}$ This is an example of a

62. See id.

63. See id.

64. Id.

65. Instant Heart Rate, AZUMIO, http://www.instantheartrate.com/android.jsp (last visited Mar. 10, 2012).

66. Id.

67. Id.

68. See Draft Guidance for Industry and FDA Staff-Pulse Oximeters - Premarket Notification Submissions [510(k)s], U.S. FOOD \& DRUG ADMIN., http://www.fda.gov/Medical 
mobile health application that is geared towards the general consumer rather than medical providers.

Another example that is still in development will allow users to give themselves eye exams with their own mobile devices. Netra, a software and add-on developed by researchers at the Massachusetts Institute of Technology ("MIT"), is an application that can provide eye exams from consumers' phones. ${ }^{69}$ The application requires an add-on piece that allows the user to look through it and will indicate if the user is nearsighted, farsighted, or has astigmatism. $^{70}$ The developers explained that the add-on costs only two dollars. They are looking to obtain FDA approval for the application. ${ }^{71}$ This example proves that companies are already aware of the possibility of regulations and are preemptively looking for approval. Nevertheless, there are many applications that have yet to seek approval likely because there is little guidance on the topic from the proper authorities.

ResolutionMD Mobile by Calgary Scientific is another mobile application marketed toward medical providers. The application allows users to view radiology images on their mobile device and will also provide capability for video consults. ${ }^{72}$ The medical professional can view CT and MR images through their device. The FDA has approved the mobile software for the iPhone and iPad but is still seeking clearance for Android devices. ${ }^{73}$ In addition, Health Canada has approved the mobile application for diagnostic use. $^{74}$ This example is unique because it has already been approved. Nonetheless, this highlights a major dividing line with mobile health applications: the software offers the ability to diagnose, yet there are many applications that are just as complex, but their developers are unsure of the need for FDA approval.

One example of such an application is the iStethoscope. The iStethoscope Pro, developed by Undercover Scientist Software, is an application used on the iPhone marketed towards the consumer. ${ }^{75}$ The user can take the iPhone, place it on their chest, and the user can record their heartbeat. ${ }^{76}$ The phone can show a phonocardiograph display of your information. ${ }^{77}$ In addition, the phone can send the recording and images of the readings to anyone

Devices/DeviceRegulationandGuidance/GuidanceDocuments/ucm071361.htm (last updated Feb. 9, 2012).

69. Eye Exam? There's An App For That: Think of Device As 'Thermometer for the Eye', THEDENVERCHANNEL.COM (Oct. 4, 2010), http://www.thedenverchannel.com/health/ 25277124/detail.html.

70. Id.

71. Id.

72. ResolutionMD Mobile, CalgaRY SCIENTIFIC INC., http://www.calgaryscientific. com/index.php?id=5 (last visited Nov. 4, 2010).

73. Id.

74. Id.

75. See iStethoscope, ITUNES PREVIEW, http://itunes.apple.com/us/app/istethoscopepro/id3221 10006? $\mathrm{mt}=8 \#$ (last visited Nov. 4, 2010).

76. Id.

77. See id. 
else through email. ${ }^{78}$ This type of application functions much like a stethoscope yet has not received any regulation of its uses.

The one capability that each of the above examples have in common is the ability to diagnose a medical purpose. On one hand, these applications could help service the world, yet these applications also represent a significant risk to the user. Consumers could download these applications and use them on their own in lieu of a medical provider at the risk that the information the consumer receives from an application might be faulty or incorrect. The bottom line is that these applications will only increase in their complexities and uses over time. Therefore, the FDA needs to issue strict guidelines that balance regulation and innovation needs.

\section{REgulation of MEdicAl DEVICES}

Mobile health applications and mobile applications in general have very little oversight. The process of bringing technology to the consumer is an issue of time. The first software developer to bring the application to market reaps the benefits of being the innovative genius that developed such an application. Up until this point, mobile applications have stayed on this path of little, if any regulation. However, with the increases in technology and innovation, applications have become more prone to regulation. In particular, mobile health applications are now being viewed and regulated as medical devices. It is necessary to take a moment to analyze the current FDA methods when evaluating what constitutes a medical device and how it should be regulated.

\section{A. Medical Device Definition by FDA}

Normally, if a product meets the FDA definition of a medical device, then the product is subject to pre and post marketing regulations by the FDA. The definition of a medical device is as follows:

an instrument, apparatus, implement, machine, contrivance, implant, in vitro reagent, or other similar or related article, including a component part, or accessory which is: recognized in the official National Formulary, or the United States Pharmacopoeia, or any supplement to them, intended for use in the diagnosis of disease or other conditions, or in the cure, mitigation, treatment, or prevention of disease, in man or other animals, or intended to affect the structure or any function of the body of man or other animals, and 
which does not achieve any of its primary intended purposes through chemical action within or on the body of man or other animals and which is not dependent upon being metabolized for the achievement of any of its primary intended purposes. ${ }^{79}$

Examples of medical devices regulated by the FDA include tongue depressors, bedpans, laser surgical devices, and x-ray machines. ${ }^{80}$ The definition itself is broad for the purposes of regulating many different devices.

The FDA's definition of a medical device is extremely broad in that it describes a medical device as "an instrument . . . intended for use in diagnosis of disease or other conditions, or in the cure, mitigation, treatment, or prevention of disease, in man or other animals. ${ }^{, 81}$ Likely many of the applications discussed previously in this Note are covered under such a broad definition. For example, some of the informational applications could be used to prevent disease or to diagnose. However, one of the most important guidelines when deciding whether these applications need FDA approval is looking to the purpose of the application. Many of the mobile health applications discussed have various intended uses, but this all depends on the particular facts of each piece of software. Overall the most fundamental aspect to understand is that the FDA has a broad definition for a purpose. The purpose requirement is to maintain safety for consumers who use these devices and to make sure that there is regulatory control in cases of defective products.

\section{B. Classes of Medical Devices Under the FDA}

Medical devices under the FDA are broken into different classes: Class I, Class II or Class III. These classes designate what amount of regulatory control is needed on the different types of medical devices. Each category represents different risks associated with the product as well as covering the varying timelines for when an application can be brought to market.

Class I devices have the least regulatory control. In most cases, these devices have a small potential for harm for the user and are simpler in design. Examples of Class I devices include bandages, examination gloves, and certain surgical instruments. ${ }^{82}$ These devices are subject to general

79. Is The Product a Medical Device?, U.S. FOOD \& DRUG ADMIN., http://www.fda.gov/MedicalDevices/DeviceRegulationandGuidance/Overview/ClassifyYour Device/ucm051512.htm (last visited Feb. 29, 2012); 21 U.S.C. § 321(h) (2010).

80. Id.

81. Id.

82. General and Special Controls, U.S. FOOD \& DRUG ADMIN., http://www.fda.gov/MedicalDevices/DeviceRegulationandGuidance/Overview/GeneralandS 
controls that include: registration of the company, device registration, and tracking of the activities of company. Moreover, devices must be manufactured under the Good Manufacturing Practices, the devices must be labeled in accordance with regulations, and the device must be submitted to premarket notification before the device can be marketed. ${ }^{83}$ However, according to the FDA, "[m]ost Class I devices are exempt from the premarket notification and/or good manufacturing practices regulation.",84

The second class of device is a Class II medical device. "Class II devices are those for which general controls alone are insufficient to assure safety and effectiveness, and existing methods are available to provide such assurances." 85 Because Class II devices require more regulation, products are subject to the general controls of Class I devices but also special controls. "Special controls may include special labeling requirements, mandatory performance standards, and postmarket surveillance." ${ }^{\text {"6 }}$ Class II devices include products such as a powered wheelchair, infusion pumps, or surgical drapes. ${ }^{87}$ This type of classification requires more time and investment on the part of the software developer or manufacturer.

Class III devices are the most regulated devices under FDA controls. "Class III devices are those for which insufficient information exists to assure safety and effectiveness solely through general or special controls." Class III devices usually sustain human life or prevent injuries or they could allow an unreasonable risk of injury. ${ }^{89}$ Because of the risks involved with these products, Class III devices require premarket approval which includes actual scientific evidence that the device is safe and effective for the products use. $^{90}$ Class III devices that require this stringent approval fall into the following categories: "(1) regulated as new drugs prior to May 28, 1976, also called transitional devices; (2) devices found not substantially equivalent to devices marketed prior to May 28, 1976; (3) Class III preamendment devices which, by regulation in $21 \mathrm{CFR}$, require a premarket approval application."91 Examples include implantable pacemaker pulse generators and endosseous implants. ${ }^{92}$

These are the three levels of regulation that a mobile health application might receive depending on whether it is found to be a medical device. Obviously all three categories require different regulation. Nevertheless, what type of regulation mobile health applications will face is still un-

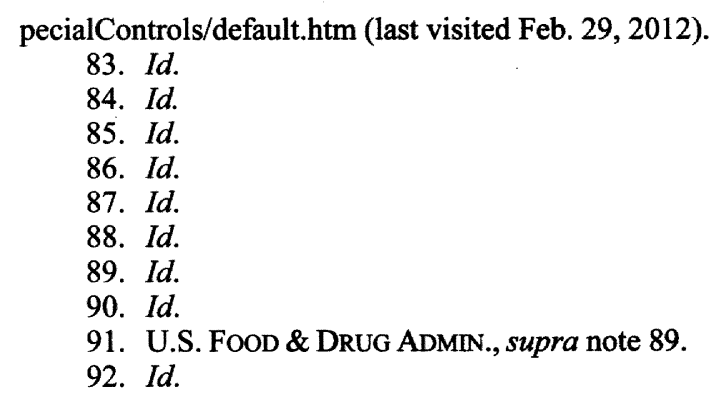


known. To complicate matters, the FDA regulates software differently depending on whether it is deemed a component or an accessory. Both definitions change how the application is regulated and who is responsible for bearing the risk of the device through the FDA regulation process:

\section{Component vs. Accessory Regulation}

Understanding if an application is a component or an accessory changes the regulation imposed by the FDA. The difference between the two can impact companies in numerous ways, and it is paramount for health software developers to understand the difference. Bradley Merril Thompson, a shareholder with the law firm Epstein Becker \& Green, explains that "[i]n the area of mobile health technology, it's important to understand that an accessory or a component of a medical device is itself a regulated medical device." ${ }^{, 93}$ Regardless of recognition, both are regulated medical devices. Companies must look toward who is purchasing the application to decide whether the device is a component or accessory.

Thompson puts it simply. "End-users buy accessories, while manufacturers buy components." 94 Of course a more in depth analysis needs to be taken, however this is a strong guidepost for analyzing this issue. The FDA wants to protect the end-user as much as possible but in the most efficient manner. The ultimate question technology companies must answer is whether the differences in components or accessories will impact their own design process.

First, the component/accessory distinction will affect the manufacturer design process. Depending on what the device is determined to be, ultimately the regulations will rest with FDA oversight or the manufacturer. Thompson explains that "[c]omponents are exempt from most FDA regulatory requirements, with the regulatory burdens being borne by the finished device manufacturer." "A5 "Accessories, on the other hand, since they go right to the end user, must meet the FDA requirements before they leave the hands of the accessory manufacturer." 96 These two requirements push the burden to different parties related to the device.

The tension is between the manufacturer of the phone and the developer of the web application. For example, if the phone manufacturer were to bear the burden of the FDA regulations, developers would not have to worry about the regulations. However, the manufacturer would likely create standards to maintain FDA regulations within their own processes. On

93. Bradley Merril Thompson, FDA Regulation of Mobile Health 3 (2010), available at http://mobihealthnews.com/wp-content/pdf/FDA_Regulation_of_Mobile_ Health.pdf.

94. Id.

95. Id.

96. Id. 
the other hand, if the application developer were required to bear the burden, then the manufacturer would not have to develop those protocols. These are the current dilemmas facing developers and manufacturers of mobile devices. Nevertheless, analysis of the current mobile health applications can help explain how some applications are classified as medical devices and whether all mobile health applications should be classified as medical devices.

\section{ARE Mobile ApPliCATIONS Medical DeVices?}

Many developers are aware of potential FDA regulations regarding their applications. However, there is very little guidance on what mobile health applications fall into the category of medical devices. Some mobile health applications have already applied for FDA approval, yet there are many developers unsure as to whether their application is applicable to the same regulatory standards. The different types of mobile health applications were discussed earlier, but this number is only increasing. Due to this problem the FDA needs to submit detailed guidance regarding the use and development of mobile health applications.

\section{A. FDA Regulation of Software}

To add some guidance to these issues, understanding the FDA guidelines regarding software in medical devices is required. The FDA has dealt with software contained in medical devices for at least a decade. Through the years the FDA has changed these guidelines in numerous ways as the agency learns more about the interaction between software and medical devices. As a result, the FDA has attempted to create guidelines that take "the least burdensome approach." ${ }^{.97}$ But, there are still substantial issues that must be dealt with when developing software for a medical device.

The FDA submitted guidelines for most software in medical devices, which includes firmware, stand-alone software applications, and accessories to medical devices. ${ }^{98}$ The FDA explains that the software referred to in its regulation of devices are those that "contain one or more software components, parts, or accessories, or are composed solely of software." 99 As a result, this definition could very likely apply to mobile health applications. Even the FDA explains that these guidelines apply to "software devices regardless of the means by which the software is delivered to the end user." 100

97. Guidance for the Content of Premarket Submissions for Software Contained in Medical Devices, U.S. FoOD \& DRUG ADMIN., http://www.fda.gov/MedicalDevices/Device RegulationandGuidance/GuidanceDocuments/ucm089543.htm (last updated May 18, 2011).

98. Id.

99. Id.

100. Id. 
The particular suggestions by the FDA regarding medical devices containing software are numerous. First, software should be labeled major, moderate, or minor. ${ }^{101}$ A major label is a software enabled device that "could directly result in death or serious injury to the patient or operator."102 A moderate label applies to a software device that could result in a minor injury. ${ }^{103}$ Lastly, a minor label applies a software device that is unlikely to cause an injury. ${ }^{104}$

The above regulations are only one method of regulating software under the level of concern method. Another method is through regulation of the software's inherent risk and intended use. According to the mHealth Regulatory Coalition, "intended uses of mHealth software can be broken into four categories: 1) display and storage; 2) transmission; 3) collection; and 4) analysis." 105 Then, the software is broken down into one of five different categories of inherent risk. ${ }^{106}$

By categorizing software into intended uses and inherent risk, the amount of FDA regulatory oversight can be ascertained. Generally, though, defining the point at which software becomes a medical device is not easy. In fact, applications that do similar actions and allow access to similar information might be regulated differently. The mHealth Regulatory Coalition explains that:

[A] scale that displays an individual's weight has an extremely low inherent risk if the individual is merely using the data for personal wellness purposes, yet the same display of the same data may have a moderate or high inherent risk if the patient is required to notify a healthcare provider when their weight reaches a certain point. ${ }^{107}$

Finally, in 2011 the FDA issued a final rule creating a new category for software called medical device data systems ("MDDS"). ${ }^{108}$ The FDA defines a MDDS as a device "that is intended to transfer, store, convert from one format to another according to preset specifications, or display medical device data ... [which] acts only as the mechanism by which med-

101. Id.

102. U.S. FoOD \& DRUG ADMIN., supra note 186.

103. Id.

104. Id.

105. Bradley Merrill Thompson et al., A Call For Clarity: Open Questions on THE SCOPE OF FDA REGULATION OF MHEALTH 37 (2010), available at http://mhealth regulatorycoalition.org/wp-content/uploads/2010/12/mrcwhitefinal122210.pdf.

106. Id.

107. Id. at 38.

108. Medical Devices; Medical Device Data Systems, 76 Fed. Reg. at 8637-8638 (Feb. 15, 2011) (to be codified at 21 C.F.R. pt. 880).. 
ical device data can be transferred, stored, converted, or displayed."109 According to this definition, it appears the FDA is limiting MDDSs to all systems or software that transfers, stores, or converts medical device data without changing the function of the connected medical device itself.

Although this definition appears clear, it does create some substantial issues for mobile health. First, if the purpose of the rule is to classify the MDDS as a Class I device, the device will be exempt from premarket notification. However, if a company is not aware its software falls under the MDDS rule, these companies will likely now be subject to registration with the FDA and other reporting and quality requirements. This assumes that the company recognizes it was subject to the MDDS rule previously or is now subject to the current standard. Nevertheless, the consequences for falling outside the MDDS classification are substantial.

For companies that were not previously subject to the MDDS classification, they may be now. This creates two distinct problems. First, a company may discover that it is no longer within the definition and is not subject to FDA Class I requirements. Second, a company may find out that it is outside the MDDS classification but still subject to FDA regulations. In short, this means that previously unregulated software may now be classified as a Class II or Class III medical device. From a larger perspective, the MDDS rule enlarged the category while also limiting the category to Class I devices.

Practically speaking, the MDDS regulation creates common issues for providers, practitioners, and developers. First, the data itself can go through multiple sources before reaching a MDDS device. A medical device can send data to device A, B, C, D, and finally reach the MDDS device in question and still be regulated. Second, health record management may fall under this classification. For example, software used for the transfer of health records to multiple healthcare organizations could be regulated. However, it is important to note that manually entered data is not considered medical device data under this rule. ${ }^{110}$ Furthermore, the MDDS classification applies to the software creator as well as manufacturers, providers, and health care organizations that choose to create their own software.

The differences between premarket approval and premarket notification are considerable from a costs perspective. For example, the fees for premarket notification in 2012 will be $\$ 4717$, whereas the costs for submitting a device for premarket approval "can reach $\$ 1,000,000$, plus user fees of an additional $\$ 185,000$ in FY 2008, increasing to $\$ 256,384$ in 2012.",11 Nonetheless, these three methods of defining when software must be regu-

109. Medical Devices; Medical Device Data System, 76 Fed. Reg. at 8638.

110. Medical Devices; Medical Device Data System, 76 Fed. Reg. at 8639.

111. Devices: General Hospital and Personal Use Devices; Reclassification of Medical Device Data System, 73 Fed. Reg. at 7502 (Feb. 8, 2008) (to be codified at 21 C.F.R. pt. 880). 
lated as a medical device creates difficulties with new mHealth companies developing mobile applications. The three FDA software guidance documents still leave considerable questions as to whether an application requires regulation and if so, what regulation is necessary.

\section{B. Bearing the Burden of Regulation}

As discussed earlier, there are many questions regarding what company is responsible for the regulations that the FDA imposes on mobile health applications. For example, the iPhone AppStore and the Android Market both have medical application categories. ${ }^{112}$ If Apple, for example, begins marketing their iPhones or iPads to physicians, this might trigger an intended use regulation for the manufacturer. ${ }^{113}$ In fact, Apple has apparently staged presentations for physicians regarding the uses of their iPad and iPhone for medical purposes. ${ }^{114}$ However, since Android is an open source software, it has not directly marketed its products to physicians simply because Android does not manufacture the phones themselves.

Another prime example is with the carriers themselves, such as AT\&T or Verizon. The mHealth Regulatory Coalition highlights the possibility of a triggering of the accessory rule. "A loss of service may implicate AT\&T, which provides wireless communication for all iPhone users." happened, AT\&T could become a regulated entity under the accessory rule due to the transmission of medical data. ${ }^{116}$ There are competing arguments on all sides. For instance, although Apple or Motorola manufactures the devices, it is the actual applications themselves performing the medical processes. Additionally, although the developers create the applications, they are merely components of the actual devices intended purposes. ${ }^{117}$ Finally, the communication companies providing wireless connectivity are already regulated devices that use the network at their own regulatory risks.

Nevertheless, Apple has already established its own way of avoiding these complex issues. As of 2009, Apple has included in their developer agreement for applications a section labeled "Regulatory Compliance for Health, Medical and Related Apps." ${ }^{118}$ In part, the clause states that the

112. Medical Category, ITUNES PREVIEW, http://itunes.apple.com/us/genre/iosmedical/id6020?mt $=8$ (last visited Oct. 31, 2010); iMedicalApps, Google Officially Launches Medical Category for Android Apps (Dec. 23, 2010), http://www.imedicalapps.com/ 2010/12/google-medical-android-apps/.

113. U.S. FOOD \& DRUG ADMIN., supra note 88.

114. Husain, supra note 57.

115. BRADLEY MERRILL THOMPSON ET AL., supra note 115, at 32.

116. Id.

117. See generally id.

118. Brian Dolan, Apple Puts Onus of FDA Clearance on iPhone Developers, MoBiHEALTHNEws (Jun. 9, 2009), http://mobihealthnews.com/2641/apple-puts-onus-of-fdaclearance-on-iphone-developers/. 
developer:

will fulfill any applicable regulatory requirements, including full compliance with all applicable laws, regulations, and policies related to the manufacturing, marketing, sale and distribution of Your Application in the United States, and in particular the requirements of the U.S. Food and Drug Administration ("FDA"), and the laws, regulations and policies of any other applicable regulatory bodies in any countries or territories where You use or make Your Application available. $^{119}$

Nonetheless it is necessary to understand the competing interests at stake regarding mHealth. For now, though, the FDA is viewing the developers of the applications as the ones responsible for bearing this burden.

\section{Current FDA Approved Mobile Applications}

Although there is little guidance strictly regarding mobile health applications, the FDA has approved mobile health applications through their current processes. One of the earliest mobile health applications to be approved was the AirStrip OB developed by AirStrip. ${ }^{120}$ The application allows physicians to remotely monitor data from the mother and child for use by OB physicians. ${ }^{121}$ The type of viewable information includes heart rates, contraction patters, as well as exam information performed by the physician. ${ }^{122}$ However, on the Apple App Store, a disclaimer explains that the application "is intended for use by Obstetricians who deliver babies." According to the FDA approval letter, the application is considered a Class II medical device and is categorized in pennatal monitoring system and accessories. ${ }^{124}$

The FDA categorizes medical devices into classifications because they must be substantially equivalent to other regulated medical devices. ${ }^{125}$

119. Id.

120. FDA Approves AirStrip's iPhone Application, SAN ANTONIO BuS. J. (Apr. 6, 2009), http://www.bizjournals.com/sanantonio/stories/2009/04/06/daily8.html.

121. Id.

122. Id.

123. AirStrip OB, ITUNES PREVIEW, http://itunes.apple.com/us/app/airstripob/id309381240? $\mathrm{mt}=8$ (last visited Dec. 31, 2010).

124. Letter from Janine M. Morris, Acting Director, Division of Reproductive, Abdominal, and Radiological Devices, U.S. Food \& Drug Admin., to Mark Job, Responsible Third Party, Regulatory Technology Services, LLC (Jan. 23, 2009), available at http://www.accessdata.fda.gov/cdrh_docs/pdf9/K090061.pdf.

125. U.S. FOOD \& DRUG ADMIN., supra note 91. 
However, if there is no similar regulated device currently available, the developer must go through premarket approval rather than premarket notification. $^{126}$ Here, AirStrip OB was found to be substantially equivalent to perinatal monitoring systems and accessories under 21 C.F.R. $\S$ 884.2740. ${ }^{127}$ Although the AirStrip OB was able to find a predicate or similar medical device already classified, many mobile health applications are so new that no other device can currently perform the same processes. One example of not having a predicate device has been with the developer of MIMvista.

MIMvista, the developer of the Mobile MIM application, received a letter from the FDA explaining that they were required to take their mobile application off the AppStore. ${ }^{128}$ Mark Cain, CTO of MIMvista, explains that the purpose of the application is to offer "full-resolution medical images, like CT, MR, or X-rays, with easy-to-use image controls such as window/level, zoom, and pan."129 On January 29, 2010 MIMvista received a letter stating that the "device has new technological characteristics that could adversely affect safety and effectiveness and raise new types of safety and effectiveness questions .... Therefore, this device is classified by statute into class III." 130 Because the application was defined as a Class III medical device, the FDA requires PMA approval or premarket approval. ${ }^{131}$ The PMA process will require clinical trials and consistent research for the application to be FDA approved. This example highlights the regulatory issues negatively impacting technological innovation.

However, in February of 2011 MIMvista was given FDA 510(k) clearance. ${ }^{132}$ The FDA explained that "the application is the first cleared by the FDA for viewing images and making medical diagnoses based on computed tomography (CT), magnetic resonance imaging (MRI), and nuclear

126. Id.

127. 21 C.F.R. $\S 884.2740$ (2012).

128. Brian Dolan, Interview: The iPhone medical app denied 510(k), MoBIHEALTHNEws (Mar. 15, 2010), http://mobihealthnews.com/6932/interview-the-iphonemedical-app-denied-510k/5/.

129. Id.

130. Greg Freiherr, FDA Fires Warning Shot Over Smart Phone Bow, DiAGNostiC IMAGING (Mar. 31, 2010), http://www.diagnosticimaging.com/news/display/article/113619/ 1547114.

131. Premarket approval (PMA) is the FDA process of scientific and regulatory review to evaluate the safety and effectiveness of Class III medical devices. Class III devices are those that support or sustain human life, are of substantial importance in preventing impairment of human health, or which present a potential, unreasonable risk of illness or injury. See Premarket Approval (PMA), U.S. Food \& DRUG ADMIN., http://www.fda.gov/ medicaldevic-

es/deviceregulationandguidance/howtomarketyourdevice/premarketsubmissions/premarketap provalpma/default.htm (last updated Jan. 24, 2012).

132. Brian Dolan, FDA clears first diagnostic radiology app, Mobile MIM, MobiHeAlthNews (Feb. 4, 2011), http://mobihealthnews.com/10173/fda-clears-firstdiagnostic-radiology-app-mobile-mim/. 
medicine technology, such as positron emission tomography (PET)."133 However, the FDA was quick to point out that "[i]t is not intended to replace full workstations and is indicated for use only when there is no access to a workstation." 134 This is just one recent issue developing with the clearances of mobile health applications and the manner in which they are used. Nonetheless, the FDA also changed their opinion on the device being a Class III. To gain $510(\mathrm{k})$ clearance the device must be classified as a Class II device. Although the device has been cleared, the clearance itself raises substantial questions of use and classification.

Current FDA regulated mobile health applications demonstrate the need for some form of regulation. However, many software developers have never had to deal with the process and have little if any information regarding their applications. For example, Cain explained that as of March 2010 it had been two years since their MobileMIM application was developed and that "[f]or tech-minded people like us, that's an incredibly long time.," ${ }^{, 135}$ Currently MobileMIM's application is available in India, Australia, Hong Kong, Singapore, and the United Kingdom. ${ }^{136}$ If the United States wants to become a world leader in a whole new industry, the FDA must develop clear guidelines to balance the interests of safety and innovation in mobile technology. In conclusion, the FDA should create cohesive guidelines to foster innovation and safety among the new mHealth industry.

\section{REGULATORY SOLUTIONS FOR MOBILE HEALTH APPLICATIONS}

Although there is a myriad of issues involved with FDA regulation of mobile health applications, it is necessary to focus on the most important questions. It is equally as important to remember that mobile health applications represent a considerable risk to users, patients, and physicians. This risk cannot go unnoticed, and the technology industry needs to recognize that regulation is not only inevitable but necessary. A balance can be reached between innovation and regulation by more clearly defining a regulatory scheme for mobile health applications.

First, is it possible to define what mobile health applications should be regulated and what applications should not be regulated? The answer is yes and no; it will change significantly with the types of innovations yet to be seen in the future. For example, twenty years ago it was tough to imagine a future where cell phones could carry and operate as mobile health assistants. Now, however, cell phones and tablets have the possibility to offer health solutions to people with very little access to health care. To begin, it is necessary to discuss what types of applications should not be regulated.

133. Id.

134. $I d$.

135. Dolan, supra note 138.

136. Id. 


\section{A. Defining Non-Regulated Mobile Health Applications}

There are two forms of mobile health applications that will help define a line between what types of applications should be regulated and what applications should not be. One example is a form of application that is merely informational. Informational applications are similar to websites in that they convey information that is readily available from other sources. These other sources could include print media, or online resources such as WebMD. Although information health applications can pose a risk to the patient or consumer, the information is to educate rather than to diagnose. Consumers may use this information for personal diagnoses, but that is not the intended use of the application.

Another form of mobile health applications that should not be regulated is lifestyle monitoring applications. Lifestyle monitoring applications include applications used for diets, weight tracking, cardiovascular logging, or even merely calorie counting. These applications allow the user to input their own person information such as height, weight, and sex to customize the results of the application. For example, MyFitnessPal, an application for tracking weight loss and fitness, is designed to be customized to the user's personal needs. ${ }^{137}$ Once the user inputs his or her height, weight, sex, and goal weight, the application returns the amount of calories needed to be consumed per day to lose that weight. ${ }^{138}$ Although applications such as these use patient information for health related reasons, they are not intended for use as a diagnosing tool but rather as a tracking tool. Most applications in this category simply allow the user to track their own data.

Although the differences in mobile health applications are subtle, to create clarity within the process, the subtleties must be analyzed. First, an application can be categorized based on the actual purposes of the application. This bridges the gap between a new method of analyzing these applications and the current method used by the FDA. ${ }^{139}$ Second, the application can then be properly placed within one of these two categories (informational or diagnostic), if the purpose matches. For example, the purpose of a mobile health application might be for inputting your health information and then the application displays medical advice. The purpose is informational; therefore, the application can then be placed within the informational category. Above all, the pertinent issue is that clarity needs to be brought to the process to spur development within this new industry. However, understanding what applications should not be regulated helps hone in on what applications should be regulated.

137. MyFitnessPaL, http://www.myfitnesspal.com/ (last visited Mar. 11, 2011).

138. Id.

139. See generally U.S. FoOD \& DRUG ADMIN., supra note 88 . 


\section{B. Defining Regulated Mobile Health Applications}

Now that certain applications can be categorized as non-regulated, the key question is whether it is possible to clearly define what mobile health applications should be regulated as medical devices. Although there is no doubt that there should be differing levels such as Class I or Class III devices, the answer is one of simplicity. To have a strict definition is not as important, at this point, as having a definition that helps developers understand when their application should be regulated as a medical device. The key answer is that any mobile health application that is not informational or lifestyle monitoring should be regulated. However simple this may be, simplicity has its benefits.

In general, with this definition comes more clarity. Right now there is no dividing line, yet, if this guideline is taken seriously, there is a definite line of what should be regulated and what should not be. For example, an application where the user inputs their height, weight, and other medical information would be characterized as lifestyle monitoring. However, an application that uses mobile technology to take your heart rate would be more than that and would need to be regulated. ${ }^{140}$ Although some form of a gray area will always exist, this at the very least creates a dividing line for developers.

The end goal for the FDA, companies, and developers should be to create a more predictable regulatory process. By simplifying the process, the FDA could create a system that is clear and predictable for developers. Additionally, the FDA would not have to risk missing certain mobile health applications simply because there is little clarity in the current process. For example, the heart monitoring application is being marketed right now but arguably could be marketed as a medical device. ${ }^{141}$ This general method of defining what mobile health applications should be regulated will allow developers to have a bright line definition to help them understand that they are not allowed to market their applications until they are approved. Unfortunately, the current FDA process and new methods have not addressed these concerns.

\section{Current FDA Position on Medical Device Review}

The FDA has faced increased scrutiny over the 501(k) medical device review process throughout the years. ${ }^{142}$ Nonetheless, recently the FDA has

140. See APPBRAIN, supra note 1.

141. See id.

142. Jaimie Oh, House Hears Arguments Over FDA's Medical Device Approval Process, BECKER's ASC REVIEW (Feb. 23, 2011), http://www.beckersasc.com/asc-supply-chainmaterials-management/house-hears-arguments-over-fdas-medical-device-approvalprocess.html. 
implemented programs to make the approval process run more efficiently in hopes of bringing medical devices to market at a faster pace while maintaining safety procedures. ${ }^{143}$ The fact that the FDA has made changes to improve the process shows that there has been and still are substantial issues impeding the medical device industry in the United States. ${ }^{144}$ Because of these issues, in 2011 the FDA released draft guidance for mobile medical applications. ${ }^{145}$

In February of 2011, the FDA announced the launch of the Medical Device Innovation Initiative. ${ }^{146}$ The Director of the FDA's Center for Devices and Radiological Health, Dr. Jeffrey Shuren, explained that the review program cover devices "that demonstrate the potential to revolutionize disease treatment, diagnosis, or health care delivery and that target unmet medical needs."147 The FDA labels the initiative as the Innovation Pathway, which gives priority to the newest medical technology and devices. ${ }^{148}$ Although the FDA has not finalized the program, the FDA has outlined several aspects to help the public understand the new program.

First, the new Innovation Pathway program itself is not drastically different than the current $510(\mathrm{k})$ or PMA regulatory paths. In fact, the FDA explained that "the Innovation Pathway is not a new regulatory pathway," but "it is a special program that gets CDRH involved earlier in the device development process." 49 At first glance, the program appears to be a newer and quicker review process, but the FDA explains that "enrollment in the Innovation Pathway would not change the scientific or regulatory standards that CDRH would use to evaluate device submissions." 150 Nevertheless, the program does purport to offer more timely and efficient reviews of medical device products. ${ }^{151}$ The FDA believes that the process will reduce review times from 300 days to approximately 150 days. ${ }^{152}$ This is significant, regardless of whether this is a new regulatory path. Nonetheless, there are still questions regarding what devices will be eligible.

143. Kathryn Foxhall, FDA Offers Simpler Path for New Medical Devices, GOVERNMENT HEALTH IT (Feb. 9, 2011), http://www.govhealthit.com/newsitem.aspx?nid= 76196.

144. Chris Kaiser, FDA and Industry Square Off Over Approval Process, CARDIOVASCULARBusiness (Feb. 24, 2011), http://www.cardiovascularbusiness.com/ index.php?option $=$ com_articles\&view $=$ article \&id $=26490 \&$ division $=$ cvb.

145. Draft Guidance for Industry and Food and Drug Administration Staff - Mobile Medical Applications, U.S. FoOD \& DRUG ADMIN. (July 21, 2011), http://www.fda.gov/ MedicalDevices/DeviceRegulationandGuidance/GuidanceDocuments/ucm263280.htm.

146. Foxhall, supra note 153.

147. Id.

148. U.S. FoOd \& DRUg ADMIN., Questions and Answers About the Medical Device Innovation Initiative (Feb. 8, 2011), http://www.fda.gov/AboutFDA/CentersOffices/ OfficeofMedicalProductsandTobacco/CDRH/CDRHInnovation/ucm242068.htm.

149. Id.

150. Id.

151. Id.

152. Id. 
The FDA is attempting to implement this process due to the drastic changes in medical device technology. Currently, the devices that would reviewed within this process are devices that "would be radically different from any legally marketed medical device in the United States in their underlying technology or manner of use."153 Additionally the device must significantly improve current treatments or diagnosis for life-threatening conditions. ${ }^{154}$ This includes a device for which there is no approved alter-. native treatment, a device that addresses an unmet public health need, or a device that addresses a national security issue. ${ }^{155}$ The requirements are broad, and likely to change, but it does not appear that mobile health applications would fit into these requirements.

Although mobile health applications contain drastically different technology, the only additional category that appears to fit would be public health. For many mobile health applications, the application addresses a need where many people do not have access to medical care. Additionally, most mobile health applications that should be regulated might not fit into that category because the technology is drastically different. Unfortunately, until the FDA chooses to address the technological changes with mobile health, the problem of clarity will not be resolved. In order to address this issue, the FDA must simplify the process for mobile health applications.

In an attempt to further clarify the categorization of mobile health applications, the FDA released draft guidance on the regulation of mobile medical applications in July of $2011 .{ }^{156}$ The document itself leaves much to be understood. Nevertheless, it appears that the FDA intends to regulate mobile medical applications when used as an accessory to a medical device. ${ }^{157}$ The document explains that "[a] mobile medical app that simply supports the intended use of a regulated medical device could be classified as [a] class I" device. ${ }^{158}$ Notably, the mobile application must not change the intended use of the medical device to which it is connected. ${ }^{159}$

In addition, the FDA intends to classify a mobile medical application in the same class as the connected medical device if the medical device "extends the intended use of the connected device."160 This example would be prevalent when a mobile application is used as an accessory to a connected medical device yet uses more detailed analysis than the connected medical device itself. In a sense, this might occur if the application is used more as a data analysis tool rather than purely informational tool. Unfortunately, if

153. Id.

154. Id.

155. $I d$.

156. $I d$.

157. $I d$.

158. Id.

159. Id.

160. $I d$. 
a mobile medical application extends the use of a Class II medical device, it would be subject to the same regulatory review.

Moreover, a mobile medical application may be subject to more stringent regulatory controls if that application creates a new intended use than that of the connected medical device. ${ }^{161}$ For example, a mobile medical application may provide diagnostic support based off of its connection to a medical device. If the connected medical device was not intended to provide that support, the FDA may regulate these applications in any class depending on the "risk posed to patient safety by the new intended use."162 Finally, mobile medical applications that allow the user to input patient specific information which is processed to "aid a clinician in making a diagnosis" will also be regulated. ${ }^{163}$

Although this guidance does provide much needed information to the industry, major issues still remain. One major issue is that, according to the FDA, all mobile medical applications belong in one category. The FDA appears to not differentiate between mobile medical applications that involve humans and those that do not. Another major issues is that, as previously discussed, intended use can be a difficult definition to pursue. Both intended use and practitioner professional use need to be evaluated for clarity in the industry. Nevertheless, one thing that is clear is that wellness mobile apps will not be subject to FDA regulation. These include apps that allow users to input diet or exercise information. Unfortunately, the FDA's current stance is limited to this document, providing little transition from the current burdensome medical device approval process.

\section{FAST TRACK SOLUTION FOR MOBILE HEALTH}

The current process, although in the midst of changes, is not a process that has ever been very amenable to the medical device industry. Although safety standards are at some of the highest levels, the industry leaders maintain that the process is hurting the industry. For example, start-up companies in the medical device sector have dropped by thirty-seven percent since $2007 .^{164}$ Although this drop can be attributed to multiple issues, there is no doubt that the current regulatory scheme plays a role in this drop. Additionally, Biosensors International, a Californian medical device company shut down its United States company last year. ${ }^{165}$ The CEO, Jeffrey B. Jump, explained that Biosensors International left because of the regulatory process and would rather spend their money abroad where processes are

161. Id.

162. Id.

163. Id.

164. Andrew Pollack, Medical Treatment, Out of Reach, N.Y. TimES (Feb. 9, 2011), http://www.nytimes.com/2011/02/10/business/10device.html?pagewanted=all.

165. Id. 
easier. ${ }^{166}$ Knowing this, the FDA has the opportunity to test a new model on technology, such as mobile health applications, that is a hybrid between the European system and the current FDA regulatory processes.

\section{A. European Model Summary}

In order to understand this hybrid model, it is helpful to analyze the European approval process in summary. The European approach and the approach in the United States have many differences. ${ }^{167}$ Although these differences bring about many issues of quality and safety, recent studies have shown smaller differences than anticipated with regards to the safety of medical devices brought to market. ${ }^{168}$ Nonetheless, the process is different and has resulted in products coming to market at a faster pace. For example, heart valves for open-heart surgery have been available in Europe since 2007, yet they will not be available in the United States until 2011 at best. ${ }^{169}$ This example is one of many that highlight the positives of the European process.

Currently, the European process is directed by the Medical Device Directive, the In-Vitro Diagnostic Directive, and the Active Implantable Medical Device Directive. ${ }^{170}$ However, each individual country has its own Competent Authority that certifies different for-profit Notified Bodies that are authorized to certify medical devices. ${ }^{171}$ In Europe, there are over seventy Notified Bodies that can authorize the use of certain medical devices. ${ }^{172}$ Once a medical device manufacturer wants to bring their device to market, they choose a Notified Body to send materials such as clinical data or literature reviews for the Notified Body to review. ${ }^{173}$ The Notified Body then performs quality assessments on the manufacturing process, and upon approval, the device has access to the European market. ${ }^{174}$ Although the process is extremely decentralized, it has resulted in similar outcomes as the FDA process with faster approval.

A recent study analyzed the recall data that is available in both the United States and Europe, finding that the numbers of recalls were identical and the types of recall were similar. ${ }^{175}$ For example, in the United States

166. Id.

167. Scott DaVIS ET AL., EU MEdical DeVICE APPROVAl SAFETY ASSESSMENT (2011), available at $\mathrm{http} / / \mathrm{www}$.advamed.org/NR/rdonlyres/061 A4AC8-D6A3-4960-826B-672214 A0A623/0/REPORTBCGEuropeanUSSafetyFINAL.pdf.

168. Id. at 1.

169. Pollack, supra note 174.

170. DAVIS ET AL., supra note 177.

171. Id.

172. Id.

173. Id.

174. $I d$.

175. Id. at 1 . 
both the recall rates for pre-market and post-market issues were nearly identical within the study. ${ }^{176}$ Generally though, the results of the study "suggest little difference between absolute number of serious recalls between the US and EU regulatory systems."177 The study even explained that "given the expectation that the EU approves more devices than the US it is likely that the EU recall rate may actually be slightly lower than the US rate."178 In conclusion, the study suggests that the overall safety argument against the European system is hindered by this data and that results are similar.

\section{B. Benefits of Separate Regulatory Scheme}

To propose an entirely new model for regulation would consume another complete article, but certain guidelines should be followed to help the transition of mobile health technology. For mobile health applications, a separate regulatory system is necessary and beneficial to the industry, consumers, and the government. There are a few important points to keep in mind regarding mobile health applications. First, mobile technology itself moves at an extremely quick pace. ${ }^{179}$ This creates problems with a regulatory process because inherently, the process itself slows the innovation related to mobile technologies more so than most other types of medical devices.

Second, access to mobile health applications is constantly at the fingertips of any person with a mobile phone. Issues with access can hardly be prevented with mobile technologies, whereas actual medical devices are rarely accessible to the consumer. For example, by 2015 , it is estimated that 500 million people will have mobile health applications on their smartphones. ${ }^{180}$ Finally, the current model does little to differentiate what mobile health applications are subject to regulatory approval. However, these issues can become catalysts for improving and implementing a new hybrid model for regulating mobile health applications as medical devices. It is because of these very issues that certain guideposts should be followed by the FDA in future implementations of regulatory schemes.

\section{A Test Model for Mobile Health Applications}

The FDA has the opportunity to drastically change its approach to

176. Id. at 7 .

177. Id. at 10 .

178. Id.

179. Brett Miller, Constantly Changing Technologies: What's a Software Developer To Do?, MASHABLE (Dec. 20, 2010), http://mashable.com/2010/12/20/constantly-changingtechnologies/.

180. Egle Mikalajunaite, $500 \mathrm{~m}$ People Will Be Using Healthcare Mobile Applications in 2015, RESEARCH2GUIDANCE (Nov. 10, 2010), http://www.research2guidance.com/500mpeople-will-be-using-healthcare-mobile-applications-in-2015/. 
medical device regulations with an industry that is still in its infancy. The proposed changes should include the following suggestions to help improve the system as a whole. In fact, the FDA needs to implement an entirely different regulatory scheme for mobile health applications. While regulating these applications as medical devices under the PMA or 501(k) process can be done more easily since the process is not changing, it is less advantageous to the industry as a whole. By maintaining the same outdated regulatory scheme, these applications and the industry are being held back. The process was not developed for such quick evolving and accessible technologies but for technologies that were evolving at a slower rate and less accessible directly to the consumer.

Second, unless the mobile health application is informational or lifestyle monitoring, the application should be regulated. If a mobile health application does not fit into one of those categories, then it must go through the regulatory process. The main reason technology companies and developers are having problems with the current process is because very little clarity exists. Developers are struggling to even understand the process. ${ }^{181}$ Here though, the definition is simple yet efficient. If an application merely relays information much like a public website, then it needs no regulatory approval from the FDA. Likewise, lifestyle monitoring applications merely allow the user to input their own information to understand how healthy they are or certain tests that may be needed. Mobile health applications beyond these two categories must face approval. By moving to a less complex definition, the FDA can bring clarity to the process.

Third, like Europe, the FDA should take a decentralized for profit approach to mobile health application approval. One of the major problems with such quick paced technology is that it is tough for even experienced developers themselves to keep pace with the changing technologies. ${ }^{182}$ The only plausible method to keep up with these changes is to leave it to private companies who are incentivized to stay up to date on the newest technologies. As mentioned previously, this decentralized method in Europe has resulted in similar recalls as have occurred the United States medical device system. ${ }^{183}$ For profit entities could register with the FDA to operate under FDA guidelines to approve these products. Generally, a similar approach can be taken to the European process. This would solve industry concerns of needing updated regulatory schemes for constantly changing technology and safety issues by allowing incentivized developers analyze and approve the products.

Finally, the FDA can require developers, companies, and application stores to prevent mobile health applications to market themselves unless

181. Miller, supra note 189.

182. Id.

183. DAVIS ET AL., supra note 177, at 10. 
approved. There is a current need for some restraint considering the analytical depth of some of these applications that are currently available for download to a consumer's phone. As mentioned earlier, if the application is merely informational or lifestyle monitoring, then it would be able to be marketed immediately. Currently, because of the lack of clarity, developers and companies are marketing these applications without knowing that they are subject to regulation. By creating penalties, application stores will be more vigilant and aware of the regulatory scheme. This will in turn influence the access factor. Right now, access is at a maximum whereas this would create a balance to safely transition consumers to use these applications. However, most applications beyond the informational and lifestyle monitoring will likely be used by practitioners.

Although these are merely guidelines and suggestions, the FDA should take this opportunity to transition into a more efficient model. By testing a new regulatory scheme on mobile health applications, the FDA will be able to balance safety and innovation properly. The FDA can solve the clarity, access, and efficiency problems merely by offering a more decentralized process for newer technologies. However, it should be noted, this Note is not advocating a complete shift in the entire process of medical devices, but offers a new model for mobile technologies. Although the mobile technology industry is just beginning, the FDA could follow these guidelines to implement a safe, efficient, and innovative regulatory policy for the United States mobile technology industry.

\section{CONCLUSION}

Regardless of the outcome, the FDA must address this issue because the mobile application industry is expanding at an alarming rate. For the United States to remain competitive, the process needs to be more streamlined so that developers within the industry know where they stand. Although there are many issues surrounding the approval of medical devices in the United States, the FDA is at a crossroads of opportunity. On one hand is the opportunity to help in the expansion of a new industry within the United States, yet on the other hand is the chance to further fuel the problems that have plagued the FDA medical device regulatory scheme. The purpose of this Note is not to propose a new standard model for FDA approval of mobile health applications. Instead this Note seeks to start a meaningful conversation which will hopefully result in advantageous results for both consumers and the industry. 